\title{
Mutational Studies of Putative Biosynthetic Genes for the Cyanobacterial Sunscreen Scytonemin in Nostoc punctiforme ATCC 29133
}

\author{
Daniela Ferreira and Ferran Garcia-Pichel* \\ School of Life Sciences, Arizona State University, Tempe, AZ, USA
}

The heterocyclic indole-alkaloid scytonemin is a sunscreen found exclusively among cyanobacteria. An 18-gene cluster is responsible for scytonemin production in Nostoc punctiforme ATCC 29133. The upstream genes scyABCDEF in the cluster are proposed to be responsible for scytonemin biosynthesis from aromatic amino acid substrates. In vitro studies of ScyA, ScyB, and ScyC proved that these enzymes indeed catalyze initial pathway reactions. Here we characterize the role of ScyD, ScyE, and ScyF, which were logically predicted to be responsible for late biosynthetic steps, in the biological

OPEN ACCESS

Edited by: Martin G. Klotz,

City University of New York, USA

Reviewed by:

Rajesh P. Rastogi,

Sardar Patel University, India

Iris Maldener,

University of Tübingen, Germany

*Correspondence:

Ferran Garcia-Pichel

ferran@asu.edu

Specialty section:

This article was submitted to Microbial Physiology and Metabolism, a section of the journal

Frontiers in Microbiology

Received: 25 January 2016 Accepted: 02 May 2016

Published: 18 May 2016

Citation:

Ferreira D and Garcia-Pichel F (2016)

Mutational Studies of Putative Biosynthetic Genes for the Cyanobacterial Sunscreen Scytonemin in Nostoc punctiforme ATCC 29133. Front. Microbiol. 7:735. doi: 10.3389/fmicb.2016.00735 context of $N$. punctiforme. In-frame deletion mutants of each were constructed $(\triangle s c y D, \Delta s c y E$, and $\triangle s c y F)$ and their phenotypes studied. Expectedly, $\Delta s c y E$ presents a scytoneminless phenotype, but no accumulation of the predicted intermediaries. Surprisingly, $\triangle s c y D$ retains scytonemin production, implying that it is not required for biosynthesis. Indeed, scyD presents an interesting evolutionary paradox: it likely originated in a duplication event from ScyE, and unlike other genes in the operon, it has not been subjected to purifying selection. This would suggest that it is a pseudogene, and yet scyD is highly conserved in the scytonemin operon of cyanobacteria. $\Delta s c y F$ also retains scytonemin production, albeit exhibiting a reduction of the production yield compared with the wild-type. This indicates that ScyF is not essential but may play an adjuvant role for scytonemin synthesis. Altogether, our findings suggest that these downstream genes are not responsible, as expected, for the late steps of scytonemin synthesis and we must look for those functions elsewhere. These findings are particularly important for biotechnological production of this sunscreen through heterologous expression of its genes in more tractable organisms.

Keywords: scytonemin, scy genes, cyanobacteria, Nostoc punctiforme, cyanobacterial deletion mutants

\section{INTRODUCTION}

In order to succeed in habitats exposed to high exposure to UV radiation, such as soil and rock surfaces, cyanobacteria must use various UV radiation defense mechanisms. Sunscreens serve as passive preventative mechanisms that allow them to intercept UV before it reaches targets in the cellular machinery (Gao and Garcia-Pichel, 2011). Scytonemin, found exclusively among 
cyanobacteria, is a brownish-yellow, lipid-soluble pigment that is excreted and accumulates in the extracellular matrix of cells exposed to UV-A radiation (315-400 nm) (Garcia-Pichel and Castenholz, 1991; Garcia-Pichel et al., 1992; Rastogi et al., 2013; Rastogi and Incharoensakdi, 2014). Structurally unique among natural products, scytonemin is a homodimeric indole-alkaloid, with a molecular mass of $544 \mathrm{~g} \mathrm{~mol}^{-1}$, composed of two heterocyclic units (cyclopentyl[b]indole) that are symmetrically connected through a carbon-carbon bond (Proteau et al., 1993) (Figure 1A). The complex ring structures allow a strong absorption in the UV-A-violet-blue range (325-425 $\mathrm{nm}$ ), with a maximum of $384 \mathrm{~nm}$ in acetone and around $370 \mathrm{~nm}$ in vivo (Garcia-Pichel and Castenholz, 1991; Proteau et al., 1993). Scytonemin has also received attention for its anti-inflammatory and anti-proliferative activities, acting as an inhibitor of human polo-like kinase 1 (Stevenson et al., 2002; Zhang et al., 2013). The increasing interest of the scytonemin use for pharmaceutical applications (Soule and Garcia-Pichel, 2014; Rastogi et al., 2015), might lead to a higher demand of this pigment. Thus, a better understanding of its biological synthetic pathway will be essential for future heterologous expression with an eye on biotechnological production, e.g., in Escherichia coli (Malla and Sommer, 2014).

Proteau et al. (1993), with the discovery of the scytonemin eight ring structure, hypothesized that this molecule was formed by the condensation of tryptophan- and phenylpropanoidderived subunits. The construction of a Nostoc punctiforme ATCC 29133 (PCC 73102) scytoneminless mutant strain, Soule et al. (2007), opened the door to the study of the molecular genetics of scytonemin biosynthesis. In order to locate the gene(s) involved in scytonemin biosynthesis, a N. punctiforme mutant unable to produce scytonemin was generated by random transposon insertion. The mutation specific site was traced to the open reading frame (ORF) Npun_R1273 (later assigned as scyD), which was part of a gene cluster involving 18 contiguous ORFs (Npun_R1276 to Npun_R1259). A closer analysis of the cluster revealed that six of these genes, scyABCDEF (Npun_R1276 to Npun_R1271), coded for novel proteins with unknown function, while the rest of genes located downstream coded for redundant orthologs of enzymes in the aromatic amino acid pathway, tryptophan and p-hydroxyphenyl-pyruvate (Soule et al., 2007, 2009b). Therefore, it was suggested that the Scy proteins were probably involved in the assembly of scytonemin from central metabolites, while the downstream region should be responsible for the delivery of biosynthetic precursors (Figure 1B). Comparative genomics studies with other strains have shown that the 18gene scytonemin cluster of $N$. punctiforme is reasonably well conserved among cyanobacteria (Sorrels et al., 2009; Soule et al., 2009b). Furthermore, transcriptional studies demonstrated that, in N. punctiforme, these genes are up-regulated under exposure to UV-A radiation (Sorrels et al., 2009; Soule et al., 2009a), and co-transcribed as a single operon (Soule et al., 2009a).

The involvement of ScyA, ScyB, and ScyC in the early stages of the scytonemin assembly was effectively demonstrated in vitro (Balskus and Walsh, 2008, 2009). ScyB (Npun_R1275), a leucine dehydrogenase homolog, catalyzes one of the first biosynthetic steps by oxidative deamination of L-tryptophan to provide indole-3 pyruvic acid (Figure 1A). TyrA (Npun_R1269), a putative prephenate dehydrogenase, is thought to be responsible for the oxidation of prephenate to p-hydroxyphenylpyruvic acid (Gao and Garcia-Pichel, 2011). Subsequently ScyA (Npun_R1276), a thiamin-dependent enzyme, mediates the acyloin coupling of indole- 3 pyruvic acid and $p$-hydroxyphenylpyruvic acid, producing the labile $\beta$-ketoacid compound 1 (Figure 1A) (Balskus and Walsh, 2008). ScyC (Npun_R1274) catalyzes the cyclization and decarboxylation of compound 1 to form a ketone (compound 2, Figure 1A). The compound yielded by ScyC is then just one oxidation state away from compound 3, a potential scytonemin monomeric precursor (Balskus and Walsh, 2009) (Figure 1A). Recently, heterologous expression of the N. punctiforme scy genes in E. coli corroborated these findings (Malla and Sommer, 2014). Upon supplementation of tryptophan and tyrosine, an E. coli BL21 strain carrying both $s c y A$ and $s c y B$ genes was able to produce a decarboxylated version of the ScyA product reported by Balskus and Walsh (2008). When $s c y C$ was expressed together with $s c y A$ and $s c y B$, an accumulation of compound 3 (together with other shunt products) was observed. Interestingly, however, the same compounds were produced when expressing all six scy genes in E. coli.

A previous study in our group predicted that the enzymes encoded by $s c y D, s c y E$, and $s c y F$ could be periplasmic proteins, and would logically be involved in the late steps of the scytonemin biosynthetic pathway (Figure 1B) (Soule et al., 2009b). Consequently, it was speculated that scytonemin biosynthesis is compartmentalized in the cell, with the monomer synthesis and initial condensation occurring in the cytoplasm, and the later reactions taking place in the periplasm, before the pigment is excreted (Soule et al., 2009b; Gao and GarciaPichel, 2011). In this study, we evaluate and discuss the role of ScyD, ScyE, and ScyF in the scytonemin biosynthetic pathway of N. punctiforme.

\section{MATERIALS AND METHODS}

\section{Cultures and Culture Conditions}

All experiments in this paper were conducted with a wildtype (WT) N. punctiforme strain ATCC 29133 (PCC 73102) derivate, UCD 153, that displays a dispersed growth under shaking culture conditions and a higher frequency of gene replacement by conjugal transfer compared to the original isolate, and mutants derived from it. WT and mutant strains of $N$. punctiforme were grown under standard culture conditions as previously described Campbell et al. (2007) in liquid Allen and Arnon medium (Allen and Arnon, 1955), diluted fourfold (AA/4), and on solidified AA medium plates. When necessary, the medium was supplemented with $2.5 \mathrm{mM} \mathrm{NH}_{4} \mathrm{Cl}$ buffered with $5 \mathrm{mM}$ MOPS ( $\mathrm{pH}$ 7.8). Neomycin at $25 \mu \mathrm{g} \mathrm{ml}^{-1}$ was used for the selection and maintenance of transformed $N$. punctiforme strains. Escherichia coli strains and derivatives were grown in liquid or solid Lysogeny Broth (LB) supplemented with 
A<smiles>CC(C)CC(C)C(=O)OCC(=O)Cc1ccc(O)cc1</smiles>

ScyA

(Acyloin condensation)<smiles>C[C@@](O)(Cc1c[nH]c2ccccc12)C(=O)Cc1ccc(O)cc1</smiles>

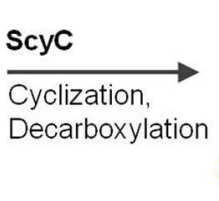<smiles>O=C1Cc2c([nH]c3ccccc23)C1Cc1ccc(O)cc1</smiles>

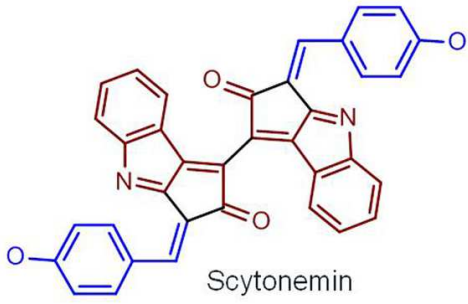

\section{SCYDEF?}

Dimerization<smiles>O=C1Cc2c([nH]c3ccccc23)/C1=C/c1ccc(O)cc1</smiles><smiles>Oc1ccc(Oc2ccco2)cc1</smiles>

B

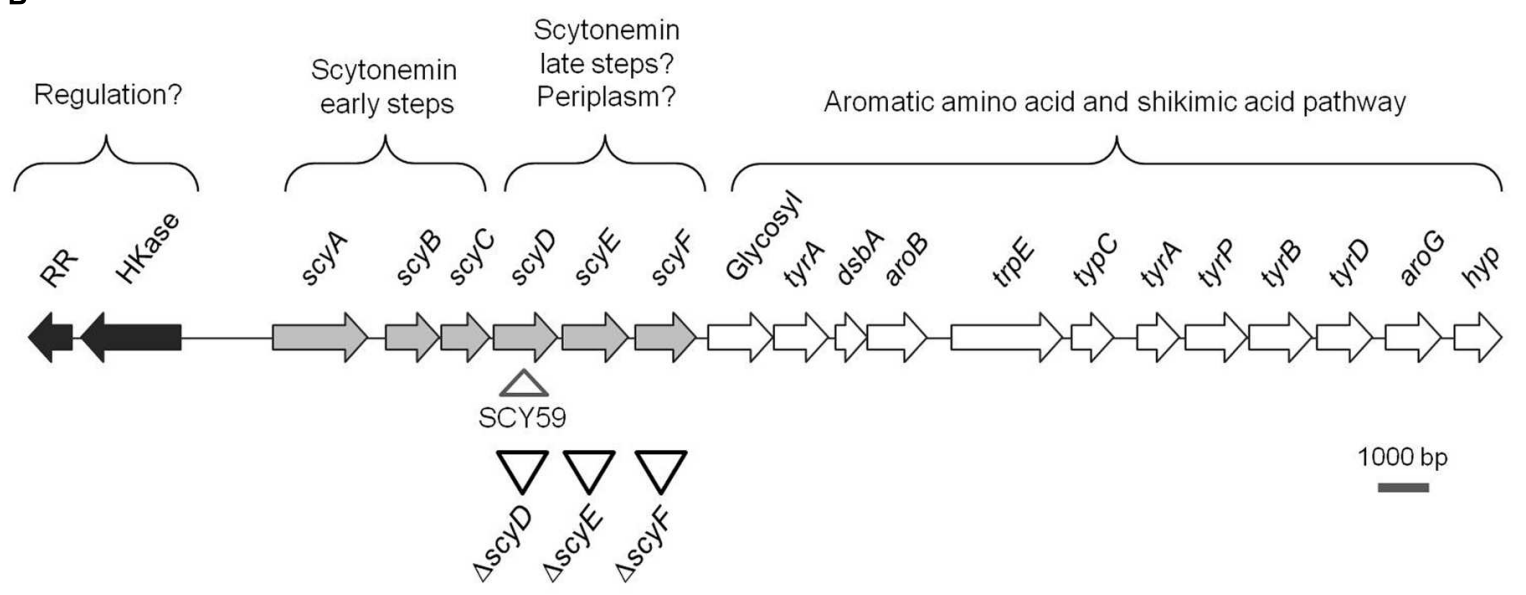

FIGURE 1 | (A) Proposed scytonemin biosynthetic pathway. The names of the enzymes (proved and speculative) involved in the process are written next to the arrows that symbolize reactions catalyzed by them. Bold numbers label the intermediate compounds. (B) Physical map of the Nostoc punctiforme genomic region containing the 18-gene cluster associated with scytonemin production. The arrows represent ORFs and are filled according to the following functional categories: white are scytonemin core genes, light gray are genes associated with aromatic amino acid biosynthesis, and dark gray are putative regulators. Upside down triangles depict approximate locations of the in-frame deletion mutants constructed in this study while the right side up triangle shows the scytoneminless insertion mutant, SCY 59, obtained previously (Soule et al., 2007) and used for comparison purposes in this study.

antibiotics at the following concentrations: kanamycin $25 \mu \mathrm{g}$ $\mathrm{ml}^{-1}$, chloramphenicol $30 \mu \mathrm{g} \mathrm{ml} \mathrm{m}^{-1}$, and ampicillin $100 \mu \mathrm{g}$ $\mathrm{ml}^{-1}$.

\section{Construction of Mutants}

All chromosomal mutations were in-frame deletions generated by PCR using $N$. punctiforme genomic DNA. Primers were designed to amplify DNA to the right and left of the deletion ( $\geq 2.0 \mathrm{~kb}$ on each side to allow for homologous recombination) with the primers adjacent to the deletion containing overlapping sequences (see Supplementary Table S1 for oligonucleotide details and gene deletions). PCR products from above were mixed and allowed to anneal via overlapping sequences, and a subsequent PCR produced a product with the deletion. These mutations should only affect the deleted portion, and not cause polar effects on downstream genes. The products were sequenced to ensure fidelity. The $s c y E$ and $s c y F$ deletions were first cloned into pUC18 and then digested with $\mathrm{XbaI}$ and Eco53kI to be subcloned into the $s a c B$-containing pRL278 (Cai and Wolk, 1990) digested with the same enzymes, while 
the scyD deletion was directly cloned into pRL278 as a XhoI fragment using restriction sites introduced on the primers. The gene deletion suicide-plasmids were introduced into WT $N$. punctiforme via triparental conjugation using E. coli strains as carriers of recombinant plasmids as described elsewhere (Cohen et al., 1994), with two exceptions: (i) no sonication was performed to fragment $N$. punctiforme filaments prior to conjugation, and (ii) conjugative plates were not supplemented with $\mathrm{CO}_{2}$ enriched air. Single recombinant strains, in which the suicideplasmid was integrated into the genome, were selected and maintained in medium containing neomycin. Strains in which the suicide-plasmid and WT gene were eliminated via a second recombination event were selected on AA plates containing 5\% (w/v) sucrose. PCRs confirmed elimination of the WT gene and replacement with the respective inactivated gene (Supplementary Figure S1).

\section{Characterization of $\Delta s c y D, \Delta s c y E$, and $\Delta$ scyF Mutant Strains}

Nostoc punctiforme biomass from WT and derived deletion mutants was tested for the ability to produce scytonemin. Cultures grown for 3-4 days, with a chlorophyll $a$ content of approximately $1-2 \mu \mathrm{g} \mathrm{ml} \mathrm{m}^{-1}$, were concentrated to $50 \mu \mathrm{g}$ of chlorophyll $a \mathrm{ml}^{-1}$ and spread over polycarbonate membrane filters $(0.4 \mu \mathrm{m}$ pore size), which were placed floating on liquid medium filled glass petri dishes, as previously described (Garcia-Pichel and Castenholz, 1991). The cells were exposed to white light $\left(7 \mathrm{~W} \mathrm{~m}^{-2}\right.$ ) supplemented with UV-A, provided by $20 \mathrm{~W}$ black-light fluorescent tubes (General Electric) at an intensity of $10 \mathrm{~W} \mathrm{~m}-2$ as described in (Garcia-Pichel et al., 1992), for a minimum of five continuous days. To ensure a correct evaluation of a scytoneminless phenotype, longer exposure times of 8 days were applied as well, and at least three experimental replicates were performed. Following UV-A exposure, the cells were harvested and the lipidsoluble pigments were extracted from whole cells in the same volume of $100 \%$ acetone. Extracts were analyzed with a spectrophotometer for absorption between 330 and $730 \mathrm{~nm}$, a strong absorption peak at $384 \mathrm{~nm}$ indicating that scytonemin had accumulated in the cells (Garcia-Pichel and Castenholz, 1991). Additionally, cultures were observed microscopically for changes in extracellular pigmentation: the presence/absence of scytonemin was easily detected by color around the cells and confirmed the spectrophotometry and HPLC data; possible colored pigment precursors were not visible (Supplementary Figure S2).

For the comparative quantification of scytonemin accumulation by the WT, $\Delta s c y D$, and $\Delta s c y F$ cells, $50 \mu \mathrm{l}$ of concentrated acetone extracts from cells exposed to UV-A for 6 days were analyzed by high pressure liquid chromatography (HPLC) using the same procedure as Karsten and Garcia-Pichel (1996). Carotenoids, chlorophyll $a$, and scytonemin were monitored in the chromatograms at $384 \mathrm{~nm}$, but spectra were also recorded continuously between 190 and $700 \mathrm{~nm}$ using an Agilent 1100 HPLC system with an online photodiode array detector. The individual pigments were identified by their characteristic absorption maxima corresponding to the appropriate retention time (chlorophyll $a$ at $17.5 \mathrm{~min}$ and scytonemin at $2.0 \mathrm{~min}$ ). Chlorophyll $a$ and scytonemin were quantified by peak areas using the integrator of the software Agilent ChemStation Rev A.06.01 (Supplementary Figure S3).

To probe for the presence of expected or novel intermediary metabolites in the mutants we implemented a dual extraction/HPLC separation protocol, followed by fraction harvesting and mass spectroscopy of candidate peaks (see Supplementary Material for details).

\section{Bioinformatic and Phylogenetic Analyses}

Orthologous sequences of cyanobacterial ScyA, ScyD, and ScyE proteins, and the respective genes coding for them, were retrieved at the NCBI (National Center for Biotechnology Information) database after Protein-Protein Basic Local Alignment Search Tool (BLASTp) analysis against the respective Scy proteins from N. punctiforme. All phylogenetic and molecular evolutionary analyses were conducted using MEGA version 6 (Tamura et al., 2013), after sequence alignment using ClustalW algorithms with the Gonnet protein weight matrix for amino acid alignments and the IUB DNA weight matrix for nucleotide alignments. Phylogenetic trees were constructed with the Minimum-Evolution, Neighbor-Joining, and MaximumLikelihood methods, and evaluated with 500 bootstrap replicates. $N$. punctiforme $\mathrm{NAD}(\mathrm{P}) \mathrm{H}$-quinone oxidoreductase subunit $\mathrm{H}$ $(\mathrm{NdhH})$ was used as the outgroup sequence. The number of non-synonymous substitutions per non-synonymous site $(d \mathrm{~N})$ and the number of synonymous substitutions per synonymous site $(d S)$ for $s c y A, s c y D$, and $s c y E$ in this dataset were calculated using different methods (see Table 1) (Jukes and Cantor, 1969; Li et al., 1985; Nei and Gojobori, 1986; Pamilo and Bianchi, 1993; Nei and Kumar, 2000).

In silico prediction of signal peptides within ScyD, ScyE, and ScyF sequences was performed using the SignalP 4.1 Server $^{1}$ (Petersen et al., 2011). Reanalysis of protein domains was carried out with the UniProt database (Universal Protein Resource), a comprehensive resource for protein sequence and annotation data (The UniProt Consortium, 2015).

${ }^{1}$ http://www.cbs.dtu.dk/services/SignalP/

TABLE 1 | Evolutionary selection analyses of scy genes among the cyanobacterial radiation, as gauged by the ratio of the number of non-synonymous substitutions per non-synonymous site $(d N)$ to the number of synonymous substitutions per synonymous site (dS), according to various algorithms.

\begin{tabular}{lccc}
\hline Algorithm & \multicolumn{3}{c}{$\boldsymbol{d N} / \boldsymbol{d}$ S Ratio } \\
\cline { 2 - 4 } & scyA & scy & scyE \\
\hline Jukes and Cantor (1969) & 0.22 & 0.97 & 0.61 \\
Li et al. (1985) & 0.24 & 0.90 & 0.57 \\
Nei and Gojobori (1986) & 0.40 & 0.99 & 0.72 \\
Nei and Kumar (2000) & 0.30 & 1.14 & 0.72 \\
Pamilo and Bianchi (1993) & 0.27 & 1.15 & 0.65
\end{tabular}




\section{RESULTS}

\section{Characterization of $\boldsymbol{N}$. punctiforme scy $D$, scyE, and scyF Knockout Mutant Strains}

In order to assess the specific role of ScyD, ScyE, and ScyF in the scytonemin biosynthetic pathway, we constructed inframe deletion mutants in which part of a gene is deleted but the rest of the operon is conserved in its proper reading frame (see Figure 1B and Supplementary Table S1 for details on construction). The DNA fragments used to generate the mutants were sequenced to guarantee that no other mutations were created during the construction. Chromosome segregation of the deletion mutants was confirmed by PCR (see Supplementary Figure S1 and Supplementary Table S2), using different combinations of oligonucleotides that flank the deleted region (so that smaller PCR products than those in the WT were expected, and observed), or that anneal to DNA regions that had been deleted (resulting in no PCR product). Examination under the optical microscope did not reveal any morphological differences, as defects in shape and size of the cells or differences in the length of the filaments, among these mutant strains grown under either white light or white light supplemented with UV-A radiation.

To determine whether or not these strains were capable of producing scytonemin, cultures of WT and its derived deletion mutants $\Delta s c y D, \Delta s c y E, \Delta s c y F$, and a previously obtained scytoneminless mutant, SCY 59 (Soule et al., 2007) were subjected to a standard test for UV-A induction (Garcia-Pichel and Castenholz, 1991), and their lipid-soluble extracts were compared by spectroscopy. Only $\Delta s c y E$ presented a scytoneminless phenotype like that known, and confirmed here, for SCY 59. Surprisingly, however, $\Delta s c y D$ and $\Delta s c y F$ extracts showed a strong absorption peak at $384 \mathrm{~nm}$, indicative of scytonemin accumulation in the cells, together with the WT (Figure 2A). HPLC analyses confirmed the identity of scytonemin by its characteristic absorption maxima corresponding to the appropriate retention time of true standards. HPLC analyses were used to quantify chlorophyll $a$ and scytonemin (Supplementary Figure S3 depicts a representative case). In addition, the inspection of UV-A induced $\Delta s c y D$ and $\Delta s c y F$ cells under the optical microscope did not reveal any differences in terms of subcellular localization of scytonemin in the mutants compared to the WT: in all three strains the pigment was clearly extracellular (Supplementary Figure S2).

Thus, among the three downstream genes in the "core biosynthetic area" of the operon, only scyE could in fact potentially code for a true biosynthetic gene in the pathway. If ScyE is responsible for picking up biosynthesis where ScyC left it, then the product of ScyC (compound 2 from Figure 1A), with molecular formula $\mathrm{C}_{18} \mathrm{H}_{15} \mathrm{NO}_{2}$ and found mass $[\mathrm{M}+\mathrm{H}]^{+}$ 278.12 (Balskus and Walsh, 2009; Malla and Sommer, 2014), should be a substrate for ScyE, and a $s c y E$ knockout mutant should accumulate this substrate under conditions of UV induction. When we performed HPLC analyses in the extracts of UV-A induced $\Delta s c y E$, we could not detect the accumulation of such compound nor of its oxidized product. We only detected the accumulation of a novel peak (Supplementary Figure S4A) with absorption maxima at ca. 198, 231, 280, and $407 \mathrm{~nm}$. However, when analyzing the collected fraction by mass spectrometry (MS), the compound was found to be $[\mathrm{M}+\mathrm{H}]^{+} \mathrm{m} / z 171.11$ (Supplementary Figure S5A), a mass quite below that expected at this point in the biosynthetic pathway.

Since the deletion of $s c y D$ and $s c y F$ did not impair scytonemin production, a comparative quantification of the scytonemin yield was performed to see if there were any subtler effects between WT and each mutant. The scytonemin/chlorophyll $a$ ratio, as measured by HPLC (Supplementary Figure S3) under identical conditions of induction, was significantly lower in $\Delta s c y F$ than in WT $(P<0.05)$, while the ratio in $\Delta s c y D$ did not differ significantly from WT (Figure 2B). These comparisons used five experimental replicates and statistical significance was determined using a Student's $t$-test (two-tailed distribution, independent, and unequal variance) to compare WT with each mutant, separately.

In fact, HPLC analyses of $\Delta s c y F$ extracts showed that, concomitantly with scytonemin production, a novel peak (Supplementary Figure S4B) with absorption maxima at ca. 210 and $370 \mathrm{~nm}$ accumulated. This fraction was collected and after MS analysis it was found to contain three distinct compounds with the following masses $[\mathrm{M}-\mathrm{H}]^{-}: 288.07,377.08$, and 417.20 (Supplementary Figure S5B).

\section{In Silico Analysis of $\boldsymbol{N}$. punctiforme ScyD, ScyE, and ScyF}

An in silico analysis of ScyD, ScyE, and ScyF amino acid sequences performed according to SignalP (Nielsen et al., 1997) was used by Soule et al. (Soule et al., 2009b) to deduce the existence of $\mathrm{N}$-terminal signal peptides in these proteins, and to postulate their periplasmic fate. An updated analyses using SignalP 4.1 (Petersen et al., 2011) confirmed the prediction of a signal peptide cleavage site in ScyD and ScyE (with Dscores of 0.794 and 0.767 , respectively, with nominal cutoff of 0.570 for significance), but not in ScyF (D-score of 0.412 with nominal cutoff of 0.510 for significance). Additionally, an evaluation at the UniProt database (2015) shows that both ScyE and ScyF contain a PEP-CTERM domain (IPR013424). This motif has been identified in a wide range of bacteria at their C-terminus and been suggested to be a protein export sorting signal (Haft et al., 2006), often associated with exopolysaccharide excretion. Thus bioinformatics still points to ScyF translocation across the membrane. Interestingly, when we examined the ScyD C-terminal region more in detail, we were able to identify a typical Proline-Glutamic acid-Proline (PEP) motif but some of the other domain characteristics seem to be missing. Our most recent analysis of the putative gene products revealed that ScyF has additional NHL repeats (IPR001258), of which a domain subgroup (IPR013017) could be involved in protein-protein interactions (Edwards et al., 2003).

\section{Phylogenetic Analysis of ScyD and ScyE}

Even though we found ScyD and ScyE to have a dissimilar involvement in scytonemin production, the two proteins are 

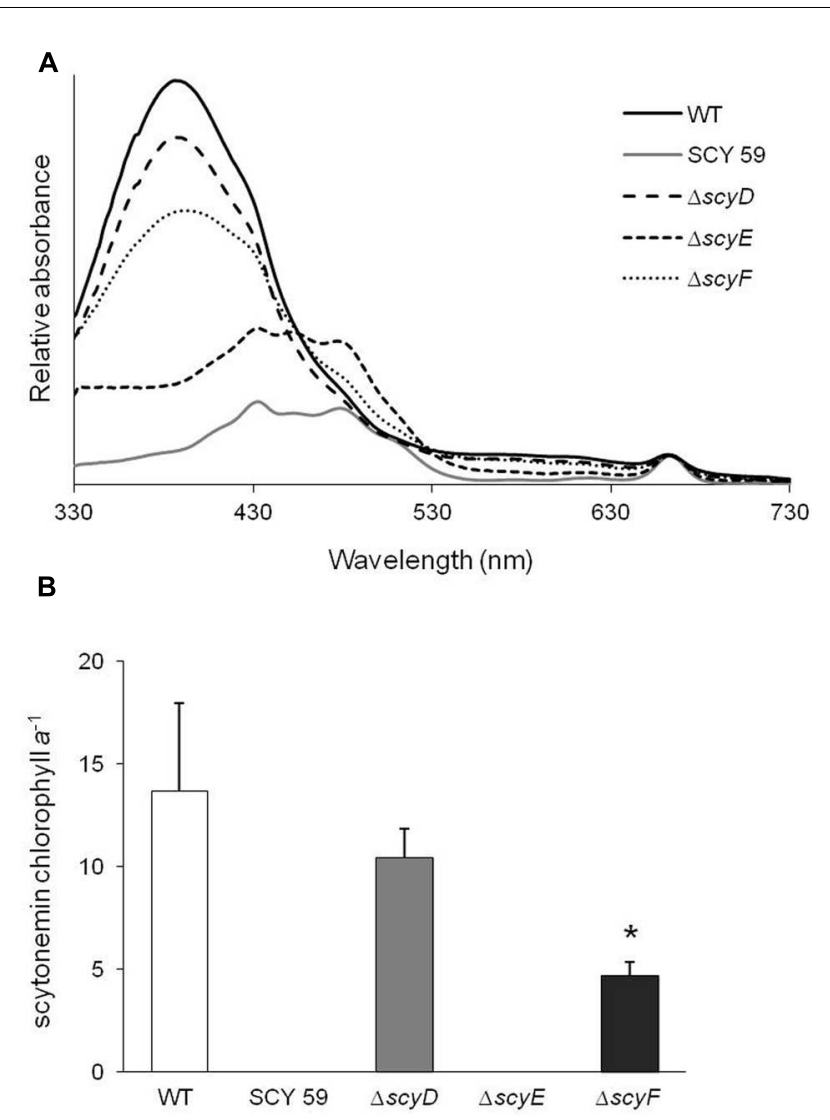

FIGURE 2 | (A) Absorption spectra of lipid-soluble pigment extracts of UV-A induced cells of $N$. punctiforme wild-type $(\mathrm{WT})$ and its derived mutants SCY 59, $\triangle s c y D, \Delta s c y E$, and $\Delta s c y F$. The characteristic peak at $384 \mathrm{~nm}$ indicates the presence of scytonemin. (B) Scytonemin to chlorophyll a ratios in extracts of WT and mutant strains after 6 days of UV-A induction, as quantified by HPLC (Supplementary Figure S3). The asterisk mark $(*)$ indicates that the scytonemin/chlorophyll a ratio in $\Delta s c y F$ is significantly different than the WT $(P<0.05)$. Error bars correspond to standard deviations from five experimental replicates.

quite similar in sequence. In fact, $s c y D$ seems to have originated as a duplication of $s c y E$ (Soule et al., 2007). Therefore, their phylogeny and molecular evolution were also investigated in more detail here. We chose cyanobacterial strains whose genomes are fully sequenced and where all six scyABCDEF genes are present. Nine strains were selected: Anabaena sp. 7120 (also known as Nostoc sp. PCC 7120), Calothrix sp. PCC 7103, Cyanothece sp. PCC 7424, Lyngbya sp. PCC 8106 (also known as Lyngbya aestuarii CCY 9616), Nodularia spumigena CCY 9414, N. punctiforme ATCC 29133 (PCC 73102), Rivularia sp. PCC 7116, Scytonema hofmanni PCC 7110 (ATCC 29171), and Tolypothrix bouteillei VB521301. Nonetheless, we realized that Cyanothece sp. PCC 7424 has two copies of ScyE instead of one of ScyE and one of ScyD; one of them shares $61 \%$ amino acid sequence identity with $N$. punctiforme ScyE (Cyanothece PCC 7424 ScyE), and the other one 44\% (Cyanothece PCC 7424 ScyE*$^{*}$. Thus, all subsequent analyses were carried out by using eight homologs of ScyD and ten of ScyE. Amino acid phylogenetic trees reconstructed using several algorithms were consistent in presenting ScyD and ScyE as part of distinct clusters (Figure 3 and Supplementary Figure S6).

We investigated nucleotide substitutions in the core genes of the scytonemin operon system to assess the degree of selection operating on each (Sorrels et al., 2009; Tai et al., 2011; Dvornyk and Jahan, 2012). We used the ratio of the number of nonsynonymous substitutions per non-synonymous site $(d N)$ and the number of synonymous substitutions per synonymous site $(d \mathrm{~S})$. If $d \mathrm{~N} / d \mathrm{~S}<1$, the gene in consideration is under purifying selection, where non-synonymous amino acid altering mutations are selected against, likely as a means to maintain function. A $d \mathrm{~N} / d \mathrm{~S}$ value around one will indicate neutral selection and a gene that is not under selective pressure. Values larger than one indicate positive (or Darwinian) selection. Purifying selection acts on the large majority of protein coding genes. In our analyses, $s c y A$, essential to scytonemin biosynthesis and previously found to be under a tight selection that does not accommodate mutational changes (Sorrels et al., 2009), was used as a control. $d \mathrm{~N} / d \mathrm{~S}$ ratios were calculated according to different algorithms (Jukes and Cantor, 1969; Li et al., 1985; Nei and Gojobori, 1986; Pamilo and Bianchi, 1993; Nei and Kumar, 2000) and found to be always well below one for $s c y A$ (ranging from 0.22 to 0.40 ) and $s c y E(0.57$ to 0.72$)$, but hovering around unity for $s c y D(0.90$ to 1.14) (Table 1), indicating that the latter is not under significant selective pressure.

\section{DISCUSSION}

Since the discovery of the operon responsible for the scytonemin production in N. punctiforme by Soule et al. (2007), advances in understanding its molecular and biochemical properties were achieved through comparative genomics, in vitro studies and heterologous expression of its genes in E. coli (Balskus and Walsh, 2008; Balskus and Walsh, 2009; Sorrels et al., 2009; Soule et al., 2009b; Malla and Sommer, 2014). Such reports helped to elucidate the role of some proteins, like ScyA, ScyB, and ScyC, or the non-housekeeping homologs of aromatic amino acids biosynthesis genes co-transcribed downstream to supplement the initial building blocks. Furthermore, these studies were essential for the formulation of some predictions, such as the putative regulatory role of the upstream response regulator and histidine kinase (Figure 1B) (Sorrels et al., 2009; Soule et al., 2009b), and the assumption that ScyD, ScyE, and ScyF are responsible for late steps in the biosynthetic pathway and, perhaps the most intriguing, that such putative enzymes, and hence the late pathway, reside in the periplasm (Soule et al., 2009b; Gao and Garcia-Pichel, 2011). The results obtained in the present study are sufficient to reject the hypothesis on the role of scyDEF.

From all in-frame deletion mutants of each gene we constructed, only $\triangle s c y E$ resulted in a scytoneminless phenotype. Clearly, neither ScyD nor ScyF are required for full scytonemin synthesis (Figure 2). It may seem paradoxical that the transposon mutagenesis effort (Soule et al., 2007) that yielded the first scytoneminless mutant, SCY 59, and pointed to this locus 


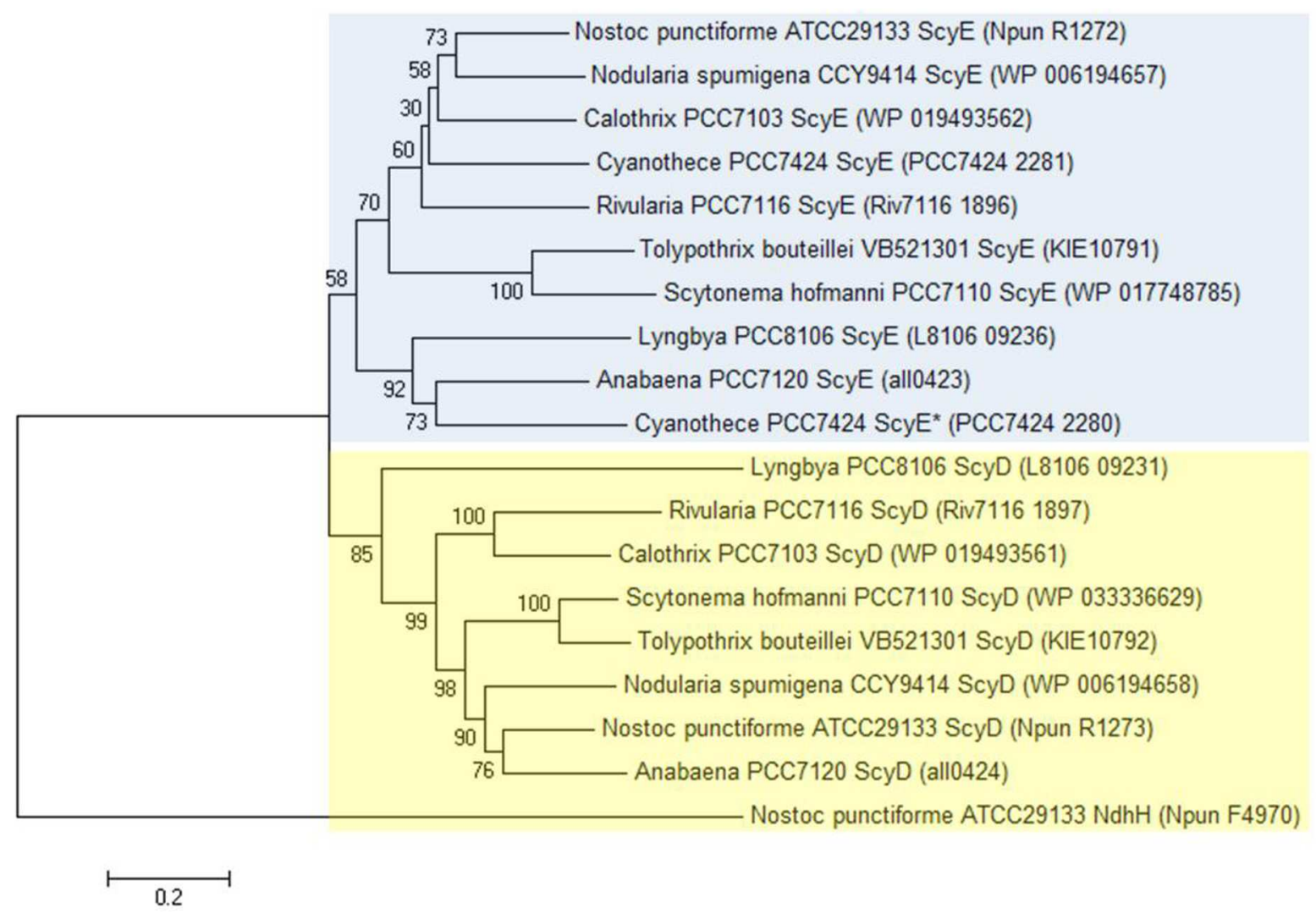

FIGURE 3 | Minimum Evolution Phylogenetic tree reconstructed on the basis of ScyD and ScyE amino acid sequences. Phylogenetic trees were created with bootstrap values from 500 replicates. N. punctiforme $\mathrm{NAD}(\mathrm{P}) \mathrm{H}$-quinone oxidoreductase subunit $\mathrm{H}(\mathrm{NdhH})$ was used as the outgroup sequence. This topology is supported by both Neighbor-Joining and Maximum-Likelihood methods (see Supplementary Figure S6). Blue box highlights the ScyE cluster, while the yellow box the ScyD. The GenBank accession number for each protein is given between brackets.

altogether, was traced to $s c y D$. However, one has to bear in mind that the insertion of the antibiotic resistance cassette in mutant SCY 59 disrupted not only the expression of $s c y D$, but also the entire set of genes downstream of it. In the light of our results, we interpret that the phenotype of SCY 59 is possibly due to a polar effect on the downstream proteins.

Nevertheless, the finding that the protein encoded by $s c y D$ does not play a role in the scytonemin biosynthesis is still puzzling due to its high conservation among cyanobacteria, where it occupies a central position in the operon (Soule et al., 2009b). In addition, two independent studies have previously shown that $s c y D$ transcript levels are also up-regulated after induction by $\mathrm{UV}-\mathrm{A}$ radiation exposure, along with the transcription of the other genes within the 18 cluster (Sorrels et al., 2009; Soule et al., 2009a). The contention that $s c y D$ might contain regulatory RNAs could be put forth, but we did not find any such predicted structures to support it. Other examples of transcription of non-functional genes (pseudogenes) that are part of functional operons in bacteria are found in the literature, possibly due to the conservation of read-through transcription as a result of their location within the operon (e.g., Forest et al., 2007; Williams et al., 2009; Feng et al., 2011). It was also clear from the phylogenetic reconstructions that $\mathrm{ScyD}$ and ScyE belong to distinct clusters (Figure 3), in agreement with the origin of one in an ancestral duplication event of the other (Soule et al., 2007) that lead to dissimilar functions. If there is indeed a differentiated function by ScyD, that function is not a requirement for scytonemin synthesis. Moreover, protein evolutionary analyses showed that, unlike the genes required for scytonemin production, scy $A$ and $s c y E, s c y D$ has not been subjected to purifying selection (Table 1), indicating that its putative translated product is not functional. In this context, we may consider $s c y D$ to be a pseudogene (Li et al., 1981; Vanin, 1985; Zheng et al., 2005), even though we must admit that such pseudogene would have been kept in place within the long evolutionary history spanning a larger portion of the cyanobacterial radiation, likely billions of years, which seems in itself paradoxical. Notwithstanding, there are some exceptions to the conservation of $s c y D$ within the scytonemin operon, for example, in Chroococcidiopsis thermalis PCC 7203 scyD (WP_015153365) is not located in the same genomic region as scyABCEF (WP_015155130 to WP_015155134), and we found Cyanothece PCC 7424 to contain two copies of $s c y E$ and none of $s c y D$. Considering all this information, we suggest that the duplication leading to $s c y D$ was redundant but it has been kept within the $s c y$ operon of most cyanobacteria due to 
its central physical location, and to the fact that it is flanked by two genes essential for scytonemin biosynthesis (scyC and $s c y E$ ).

As expected, $\triangle s c y E$ presented a scytoneminless phenotype (Figure 2) but none of its potential substrates seem to accumulate in significant quantities. In particular, no accumulation of the ketone catalyzed by ScyC (compound 2 in Figure 1A, $[\mathrm{M}+\mathrm{H}]^{+}$ $\mathrm{m} / z$ 278.12), nor its potential oxidized product, the monomer moiety of scytonemin (compound 3 in Figure 1A, $[\mathrm{M}+\mathrm{H}]^{+}$ $m / z$ 276.10) (Malla and Sommer, 2014), were detected. However, we did distinguish the accumulation of small quantities of a $\Delta s c y E$ exclusive compound with $[\mathrm{M}+\mathrm{H}]^{+} m / z 171.11$, a mass much smaller than expected. We cannot exclude the possibility that compound 2 is toxic to $N$. punctiforme's cells, thus being quickly degraded into the compound found $[\mathrm{M}+\mathrm{H}]^{+}$171.11, though this would be quite unlikely because an oxidized version of compound 3 is found in Nostoc spp. strains (Kobayashi et al., 1994; Ploutno and Carmeli, 2001). Consistent with our findings, Malla and Sommer (2014) showed that, upon supplementation of tryptophan and tyrosine, E. coli produces the same monomer moiety of scytonemin when expressing either $s c y A B C$ or $s c y A B C D E F$. Altogether, these data confirm that the $s c y E$ position in the operon does not mirror the biosynthetic sequence of reactions ( $\mathrm{ScyE}$ is not involved in the oxidation of the ScyC product), and indicate that ScyE is not involved in the last steps of scytonemin biosynthesis in a direct manner. Unfortunately, in silico analysis of ScyE conserved domains does not offer clear suggestions as to its role, as it is part of the beta-propeller clan, a protein family that is functionally quite heterogeneous. ScyE can be predicted to be periplasmic on the basis of the presence of putative signal peptides (Soule et al., 2007, 2009b; this study). It has been shown that cyanobacteria can export proteins across the cytoplasmic membrane through the Sec and the Tat pathways using such signal peptides (Liberton and Pakrasi, 2008; Barnet et al., 2011). SignalP 4.1 (Petersen et al., 2011), supports the prediction of a ScyE translocation through the Sec system. The exact role of ScyE, while crucial for scytonemin biosynthesis, remains to be established.

$\triangle s c y F$ showed a reduction of the sunscreen yield compared with the WT and it accumulated an uncharacterized product only in cultures exposed to UV-A radiation (Figure 2B and Supplementary Figure S4B). This suggests that ScyF is not essential but might be an adjuvant for the scytonemin synthesis. A UniProt database (2015) analysis shows that the ScyF amino acid sequence contains a NHL domain subgroup (IPR013017) that could be involved in proteinprotein interactions (Edwards et al., 2003). We can speculate that ScyF is a chaperone that helps the folding of an enzyme involved in the scytonemin synthetic pathway. On the other hand, it is also possible that ScyF is the catalyst of an otherwise spontaneous reaction, or that it suppresses the formation of an off-pathway reaction, in a manner similar to ScyC, which steers the pathway away from a non-enzymatic decarboxylation of the ScyA product, that would result in the production of an $\alpha$-hydroxyketone regioisomer (Balskus and Walsh, 2009).

\section{CONCLUSION}

Our findings suggest that the proteins encoded by $s c y D E F$, the region downstream of the known scytonemin biosynthetic core scy $A B C$, are not, as predicted, responsible for the late reactions in the biosynthetic pathway. It is clear that ScyA, ScyB, and ScyC are not sufficient for the pigment biosynthesis. Therefore, one must look elsewhere in the genome for the missing late enzymes. A comparative genomics study discovered that in most cyanobacteria the scytonemin operon contains a group of five genes of unknown function and high phylogenetic conservation, placed typically immediately downstream of the $s c y D E F$ region (Soule et al., 2009b). In N. punctiforme and closely related strains these genes are also present, but located outside of the main operon as a satellite five-gene cluster (Npun_F5232 to Npun_F5236). We consider these to be good candidates, and will be investigating their possible involvement in the near future.

\section{AUTHOR CONTRIBUTIONS}

FG-P conceived this study; DF and FG-P designed the experiments; DF performed the experimental work; manuscript was written by DF with help by FG-P. All authors have read and approved the final manuscript.

\section{FUNDING}

This work was funded by NSF grant 1158551 to FGP.

\section{ACKNOWLEDGMENTS}

We would like to thank Jack Meeks and Elsie Campbell for providing the wild-type $N$. punctiforme strain UCD 153 and the plasmid pRL278 used for chromosome mutations, Demetra Hamill for technical support, Blake Dirks for N. punctiforme photomicrographs, and Daniel Brune for HPLC and MS assistance.

\section{SUPPLEMENTARY MATERIAL}

The Supplementary Material for this article can be found online at: http://journal.frontiersin.org/article/10.3389/fmicb. 2016.00735 


\section{REFERENCES}

Allen, M. B., and Arnon, D. I. (1955). Studies on nitrogen-fixing blue-green algae. I. Growth and nitrogen fixation by Anabaena cylindrical Lemm. Plant Physiol. 30, 366-372. doi: 10.1104/pp.30.4.366

Balskus, E. P., and Walsh, C. T. (2008). Investigating the initial steps in the biosynthesis of cyanobacterial sunscreen scytonemin. J. Am. Chem. Soc. 130, 15260-15261. doi: 10.1021/ja807192u

Balskus, E. P., and Walsh, C. T. (2009). An enzymatic cyclopentyl[b]indole formation involved in scytonemin biosynthesis. J. Am. Chem. Soc. 131, 1464814649. doi: 10.1021/ja906752u

Barnet, J. P., Robinson, C., Scanlan, D. J., and Blindauer, C. A. (2011). The Tat protein export pathway and its role in cyanobacterial metalloprotein biosynthesis. FEMS Microbiol. Lett. 325, 1-9. doi: 10.1111/j.1574-6968.2011.02391.x

Cai, Y. P., and Wolk, C. P. (1990). Use of a conditionally lethal gene in Anabaena sp. strain PCC 7120 to select for double recombinants and to entrap insertion sequences. J. Bacteriol. 172, 3138-3145.

Campbell, E. L., Summers, M. L., Christman, H., Martin, M. E., and Meeks, J. C. (2007). Global gene expression patterns of Nostoc punctiforme in steadystate dinitrogen-grown heterocyst-containing cultures and at single time points during the differentiation of akinetes and hormogonia. J. Bacteriol. 189, 52475256. doi: 10.1128/JB.00360-07

Cohen, M. F., Wallis, J. G., Campbell, E. L., and Meeks, J. C. (1994). Transposon mutagenesis of Nostoc sp. strain ATCC 29133, a filamentous cyanobacterium with multiple cellular differentiation alternatives. Microbiology 140, 3233-3240. doi: 10.1099/13500872-140-12-3233

Dvornyk, V., and Jahan, A. S. (2012). Extreme conservation and nonneutral evolution of the cpmA circadian locus in a globally distributed Chroococcidiopsis sp. from naturally stressful habitats. Mol. Biol. Evol. 29, 3899-3907. doi: 10.1093/molbev/mss191

Edwards, T. A., Wilkinson, B. D., Wharton, R. P., and Aggarwal, A. K. (2003). Model of the brain tumor-Pumilio translation repressor complex. Genes Dev. 17, 2508-2513. doi: 10.1101/gad.1119403

Feng, Y., Chen, Z., and Liu, S.-L. (2011). Gene decay in Shigella as an incipient stage of host-adaptation. PLoS ONE 6:e27754. doi: 10.1371/journal.pone.0027754

Forest, C., Faucher, S. P., Poirier, K., Houle, S., Dozois, C. M., and Daigle, F. (2007). Contribution of the stg fimbrial operon of Salmonella enterica serovar Typhi during interaction with human cells. Infect. Immun. 75, 5264-5271. doi: 10.1128/IAI.00674-07

Gao, Q., and Garcia-Pichel, F. (2011). Microbial ultraviolet sunscreens. Nat. Rev. Microbiol. 9, 791-802. doi: 10.1038/nrmicro2649

Garcia-Pichel, F., and Castenholz, R. W. (1991). Characterization and biological implications of scytonemin, a cyanobacterial sheath pigment. J. Phycol. 27, 395-409. doi: 10.1111/j.0022-3646.1991.00395.x

Garcia-Pichel, F., Sherry, N. D., and Castenholz, R. W. (1992). Evidence for an ultraviolet sunscreen role of the extracellular pigment scytonemin in the terrestrial cyanobacterium Chlorogloeopsis sp. Photochem. Photobiol. 56, 17-23. doi: 10.1111/j.1751-1097.1992.tb09596.x

Haft, D. H., Paulsen, I. T., Ward, N., and Selengut, J. D. (2006). Exopolysaccharideassociated protein sorting in environmental organisms: the PEP-CTERM/EpsH system. Application of a novel phylogenetic profiling heuristic. BMC Biol. 4:29. doi: 10.1186/1741-7007-4-29

Jukes, T. H., and Cantor, C. R. (1969). "Evolution of protein molecules," in Mammalian Protein Metabolism, ed. H. N. Munro (New York, NY: Academic Press), 21-132.

Karsten, U., and Garcia-Pichel, F. (1996). Carotenoids and mycosporine-like amino acid compounds in members of the genus Microcoelus (Cyanobacteria): a chemosystematic study. Syst. Appl. Microbiol. 19, 285-294. doi: 10.1016/S07232020(96)80054-3

Kobayashi, A., Kajiyama, S., Inawaka, K., Kanzaki, H., and Kawazu, K. (1994). Nostodione A, a novel mitotic spindle poison from a blue-green alga Nostoc commune. Z. Naturforsch. C 49, 464-470.

Li, W., Wu, C., and Luo, C. (1985). A new method for estimating synonymous and nonsynonymous rates of nucleotide substitution considering the relative likelihood of nucleotide and codon changes. Mol. Biol. Evol. 2, 150-174.

Li, W. H., Gojobori, T., and Nei, M. (1981). Pseudogenes as a paradigm of neutral evolution. Nature 292, 237-239. doi: 10.1038/292237a0
Liberton, M., and Pakrasi, H. B. (2008). "Membrane systems in cyanobacteria," in The Cyanobacteria, eds A. Herrero and E. Flores (Norfolk: Caister Academic Press), 271-288.

Malla, S., and Sommer, M. O. A. (2014). A sustainable route to produce the scytonemin precursor using Escherichia coli. Green Chem. 16, 3255-3265. doi: 10.1039/C4GC00118D

Nei, M., and Gojobori, T. (1986). Simple methods for estimating the numbers of synonymous and nonsynonymous nucleotide substitutions. Mol. Biol. Evol. 3 , $418-426$.

Nei, M., and Kumar, S. (2000). Molecular Evolution and Phylogenetics. New York, NY: Oxford University Press.

Nielsen, H., Engelbrecht, J., Brunak, S., and von Heijne, G. (1997). Identification of prokaryotic and eukaryotic signal peptides and prediction of their cleavage sites. Protein Eng. 10, 1-6. doi: 10.1093/protein/10.1.1

Pamilo, P., and Bianchi, N. O. (1993). Evolution of the Zfx and Zfy genes: rates and interdependence between the genes. Mol. Biol. Evol. 10, 271-281.

Petersen, T. N., Brunak, S., Heijne, G., and Nielsen, H. (2011). SignalP 4.0: discriminating signal peptides from transmembrane regions. Nat. Methods 8, 785-786. doi: 10.1038/nmeth.1701

Ploutno, A., and Carmeli, S. (2001). Prenostodione, a novel UV-absorbing metabolite from a natural bloom of the cyanobacterium Nostoc species. J. Nat. Prod. 64, 544-545. doi: 10.1021/np000562w

Proteau, P. J., Gerwick, W. H., Garcia-Pichel, F., and Castenholz, R. (1993). The structure of scytonemin, an ultraviolet sunscreen pigment from the sheaths of cyanobacteria. Experientia 49, 825-829. doi: 10.1007/BF01923559

Rastogi, R. P., and Incharoensakdi, A. (2014). Characterization of UVscreening compounds, mycosporine-like amino acids, and scytonemin in the cyanobacterium Lyngbya sp. CU2555. FEMS Microbiol. Ecol. 87, 244-256. doi: 10.1111/1574-6941.12220

Rastogi, R. P., Sinha, R. P., and Incharoensakdi, A. (2013). Partial characterization, UV-induction and photoprotective function of sunscreen pigment, scytonemin from Rivularia sp. HKAR-4. Chemosphere 93, 1874-1878. doi: 10.1016/j.chemosphere.2013.06.057

Rastogi, R. P., Sonani, R. R., and Madamwar, D. (2015). Cyanobacterial sunscreen scytonemin: role in photoprotection and biomedical research. Appl. Biochem. Biotechnol. 176, 1551-1563. doi: 10.1007/s12010-015-1676-1

Sorrels, C. M., Proteau, P. J., and Gerwick, W. H. (2009). Organization, evolution, and expression analysis of the biosynthetic gene cluster for scytonemin, a cyanobacterial UV-absorbing pigment. Appl. Environ. Microbiol. 75, 48614869. doi: 10.1128/AEM.02508-08

Soule, T., and Garcia-Pichel, F. (2014). "Ultraviolet photoprotective compounds from cyanobacteria in biomedical applications," in Cyanobacteria: An Economic Perspective, eds N. K. Sharma, A. K. Rai, and L. J. Stal (Chichester: John Wiley \& Sons, Ltd.), 119-143.

Soule, T., Garcia-Pichel, F., and Stout, V. (2009a). Gene expression patterns associated with the biosynthesis of the sunscreen scytonemin in Nostoc punctiforme ATCC 29133 in response to UVA radiation. J. Bacteriol. 191, 4639-4646. doi: 10.1128/JB.00134-09

Soule, T., Palmer, K., Gao, Q., Potrafka, R. M., Stout, V., and Garcia-Pichel, F. (2009b). A comparative genomics approach to understanding the biosynthesis of the sunscreen scytonemin in cyanobacteria. BMC Genomics 10:336. doi: 10.1186/1471-2164-10-336

Soule, T., Stout, V., Swingley, W. D., Meeks, J. C., and Garcia-Pichel, F. (2007). Molecular genetics and genomic analysis of scytonemin biosynthesis in Nostoc punctiforme ATCC 29133. J. Bacteriol. 189, 4465-4472. doi: 10.1128/JB. 01816-06

Stevenson, C. S., Capper, E. A., Roshak, A. K., Marquez, B., Grace, K., Gerwick, W. H., et al. (2002). Scytonemin - a marine natural product inhibitor of kinases key in hyperproliferative inflammatory diseases. Inflamm. Res. 51, 112-114. doi: 10.1007/BF02684014

Tai, V., Poon, A. F. Y., Paulsen, I. T., and Palenik, B. (2011). Selection in coastal Synechococcus (cyanobacteria) populations evaluated from environmental metagenomes. PLoS ONE 6:e24249. doi: 10.1371/journal.pone.0024249

Tamura, K., Stecher, G., Peterson, D., Filipski, A., and Kumar, S. (2013). MEGA6: molecular evolutionary genetics analysis version 6.0. Mol. Biol. Evol. 30, 27252729. doi: $10.1093 / \mathrm{molbev} / \mathrm{mst} 197$

The UniProt Consortium (2015). UniProt: a hub for protein information. Nucleic Acids Res. 43, D204-D212. doi: 10.1093/nar/gku989 
Vanin, E. F. (1985). Processed pseudogenes: characteristics and evolution. Annu. Rev. Genet. 19, 253-272. doi: 10.1146/annurev.ge.19.120185.001345

Williams, D. L., Slayden, R. A., Amin, A., Martinez, A. N., Pittman, T. L., Mira, A., et al. (2009). Implications of high level pseudogene transcription in Mycobacterium leprae. BMC Genomics 10:397. doi: 10.1186/1471-2164-10-397

Zhang, G., Zhang, Z., and Liu, Z. (2013). Scytonemin inhibits cell proliferation and arrests cell cycle through downregulating Plk1 activity in multiple myeloma cells. Tumor Biol. 34, 2241-2247. doi: 10.1007/s13277-013-0764-5

Zheng, D., Zhang, Z., Harrison, P. M., Karro, J., Carriero, N., and Gerstein, M. (2005). Integrated pseudogene annotation for human chromosome 22: evidence for transcription. J. Mol. Biol. 349, 27-45. doi: 10.1016/j.jmb.2005.02.072
Conflict of Interest Statement: The authors declare that the research was conducted in the absence of any commercial or financial relationships that could be construed as a potential conflict of interest.

Copyright (c) 2016 Ferreira and Garcia-Pichel. This is an open-access article distributed under the terms of the Creative Commons Attribution License (CC BY). The use, distribution or reproduction in other forums is permitted, provided the original author(s) or licensor are credited and that the original publication in this journal is cited, in accordance with accepted academic practice. No use, distribution or reproduction is permitted which does not comply with these terms. 